\title{
J-Mirror, Magnetic Bottle Using the Longitudinal Invariant of Motion
}

J-Mirror, Magnetic Bottle Using the Longitudinal Invariant of Motion

T. Ohkawa

Gulf General Atomic Incorporated

John Jay Hopkins Laboratory for Pure and Applied Science San Diego, California 92112

A magnetic bottle may be obtained by having a maximum of longitudinal invariant $(J)$ in both the radial and the axial direction. The "loss disc" in this case is the region in the velocity space corresponding to small parallel velocities or small values of the invariant. Since the excursion of the orbit with a small $J$ is confined to a small volume, the collisions only in this volume will lead to the loss of the particle. Consequently, the confinement time limited by collisions is much longer than that of the $\mu$-mirror configuration. On the other hand, the constancy of $J$ may be more readily destroyed by nonadiabaticity or low frequency electric field. 
INTRODUCTION

The magnetic mirror device utilizes the constancy of the magnetic moment $(\mu)$ to confine the particle orbits. A magnetic bottle may similarly be obtained by using the constancy of the longitudinal invariant $J$ defined by $J=m \oint v_{\|} d s$. If a magnetic configuration is designed such that $J$ of a typical orbit decreases away from the confining region, (maximum $J$ configuration) the particle orbits with $J$ larger than a critical value are confined. In the following the advantages and the disadvantages of these configurations are compared with the ordinary mirror configuration.

\section{MAXIMUM J CONFIGURATION}

We shall take a linear quadrupole configuration as an example. The magnetic moment $\mu$ and the longitudinal invariant $J$ are assumed to be constant of motion. This may be a poor approximation near the separatrix.

We shall use customary $\psi$ (flux function) and $X$ (magnetic potential) coordinates. The longitudinal invariant is defined by

$$
J=m \oint \frac{\dot{x}}{B^{2}} d x
$$

It is equal to the area enclosed by the trajectory in the phase space for the trapped particles or by the trajectory and the periodic boundary of $x$ for the untrapped particles. 
J-Mirror, Magnetic Bottle Using the Longitudinal

Invariant of Motion

Since $\mu$ is a constant of motion, Eq. (1) may be written as

$$
J(v, \psi, \mu)=\int \sqrt{\mathrm{mv}^{2}-2 \mu B(\psi, x)}(d x / B)
$$

For a given $\mathrm{v}$ and $\psi$, the limiting values of $\mathrm{J}$ are:

(a) particles without parallel velocity (maximum $\mu$ ), $J=0$ and

(b) particles without perpendicular velocity $(\mu=0)$,

$$
J=m v \oint(d x / B)=m v \oint d s
$$

Therefore, the necessary condition for a maximum $J$ 'configuration is that $\oint \mathrm{ds}$ has a maximum. The particles with $\mathrm{J}=0$ are in the "loss disc", because $J=0$ orbits cannot be contained by maximum $J$ configuration.

For an intermediate value of $\mathrm{J}, \mathrm{Eq}$. (2) is differentiated with respect $\psi$,

$$
\frac{\partial J}{\partial \psi}=-\oint\left(m v^{2}-2 \mu B\right)^{-\frac{1}{2}}\left(m v^{2}-\mu B\right) B^{-2}(\partial B / \partial \psi) d X
$$

It may be rewritten as

$$
\frac{\partial J}{\partial \psi}=-m \oint\left(v_{\|}^{2}+v_{\perp}^{2} / 2\right)\left(I / B R_{c}\right)\left(d s / v_{\|}\right)=-\left(\frac{e}{c}\right) \oint v_{g}\left(d s / v_{\|}\right)
$$

where $R_{c}^{-1}$ is the curvature of the line and $v_{g}$ is the drift velocity. Equation (5) shows that the condition for a maximum $J$ configuration is to have all particles drift, averaged over the orbits, in the good direction For the particles which have the turning point at the maximum in $x$ direction of the magnetic field strength, the integral is determined by the local value of the integrand. Therefore a maximum $J$ configuration has to 


\section{Ohkawa}

have the minimum $B$ in $\psi$ direction locally at the maximum in the $x$ direction. This has been suggested first by M. Rosenbluth ${ }^{(1)}$ to stabilize the residual flute instability.

The maximum $J$ region is defined by

$$
\int\left(m v^{2}-2 \mu B\right)^{-\frac{1}{2}}\left(m v^{2}-\mu B\right) B^{-2}(\partial B / \partial \psi) d X>0
$$

for all values of $\mu$ between 0 and the maximum value given by $\left(\mathrm{mv}^{2} / 2 \mathrm{~B}_{\mathrm{m}}\right)$ where $B_{m}$ is the magnetic field strength at the minimum on a flux line. $J$ may be made to have maximum in the axial direction by bringing current carrying rods of a linear multipole closer at the both ends. The magnetic field configuration is compressed at both the ends. The line length is reduced proportionally with the distance between the axis and the rods. The magnetic field increases proportionally. $J$ for a given $\mathrm{v}, \mu$ and $\psi$ decrease towards the ends of the linear multipole. The maximum value of $J$ at the end is given by

$$
J^{*}=m v \int_{\text {on the separatrix }} \text {. }
$$

The orbits having $J>J^{*}$ are confined in the $J$-mirror. The orbits drift in the axial direction as it approaches towards the separatrix. After it crosses the separatrix, it reverses the direction of the axial drift and moves away from the separatrix. The orbits are furthest from the separatrix at the center of the J-mirror.

The orbits with $\mathrm{J}<\mathrm{J}^{*}$ escape from the $\mathrm{J}$-mirror. However, they are confined in a region near the minimum of the magnetic field on a flux line. This is the important property making the collisions between particles outside of this region irrelevant to the confinement time. 


\section{J-Mirror, Magnetic Bottle Using the Longitudinal Invariant of Motion}

\section{LOSS DUE TO COLLISIONS}

If we denote the volume of the region containing the orbits with $J<J^{*} \quad V_{1}$ and the total volume $V_{0}$, the confinement time $T$ is given by

$$
\tau=\left(V_{0} / V_{1}\right) T_{C}
$$

where $\tau_{c}$ is the collision time as in the ordinary $\mu$-mirror. The ratio $\mathrm{V}_{0} / \mathrm{V}_{1}$ may be $5 \sim 10$. The improvement of this order over the $\mu$-mirror is significant, because the $\mu$-mirror loss-rate is barely tolerable.

\section{STABILITY}

The plasma will be stable against the low-frequency mode like residual flute mode, because all the particles are drifting in good direction. The source of the instability is the reduced density along the line of force near the minimum of the magnetic field, or the "loss-disc". There may be a mode near the bouncing frequency of ions filling the loss-disc.

\section{NON-ADIABACITY}

The largest problem is probably the non-adiabacity of the motion near the separatrix. The maximum $J$ region is near the separatrix and the orbits 


\section{Ohkawa}

have to cross the separatrix before it reverses the axial direction. The further calcilation, digital if necessary, is needed to test this point.

\section{COLLISIONAL CASE}

If collisions are frequent, the plasma will flow out from the ends. The flow velocity is given by $E / B$, if it is due to $E \times B$ drift, or by $\mathrm{T} / \mathrm{BR}_{\mathrm{C}}$, if due to $\nabla \mathrm{B}$ drift. In either case, the velocity $\mathrm{v}_{f}$ is given by

$$
\mathrm{v}_{\mathrm{f}}=\frac{1}{\alpha} \frac{\mathrm{T}}{\mathrm{rB}}
$$

where $\alpha$ is a numerical constant and it is assumed $E \propto T / r$ and $R_{c} \propto r$. Then the confinement time $\tau_{e}$ limited by the end loss is given by

$$
\tau_{e}=\frac{\alpha B_{2} r_{2} I}{T}\left(\frac{r_{1}^{2}}{r_{2}^{2}}\right)
$$

where $r_{1}$ and $r_{2}$ are the radii of the device and $B_{2}$ and $B_{1}$ are the magnetic field strength at the center and at the ends, and $I$ is the length of the device. The confinement time due to radial loss $\tau_{r}$. may be given by

$$
\tau_{r}=\alpha^{\prime} B_{1} r_{I}^{2} / T
$$

where a Bohm type diffusion loss is assumed. 


\section{J-Mirror, Magnetic Bottle Using the Longitudinal} Invariant of Motion

For these two to be equal, we have

$$
\alpha \frac{B_{2}}{B_{1}}\left(\frac{L}{r_{2}}\right) \approx \alpha^{\prime}
$$

Since $\alpha^{\prime}$ larger than 1500 is preferred, the condition for a tolerable end loss is

$$
\alpha \cdot\left(\frac{L}{r_{1}}\right)\left(\frac{r_{1}}{r_{2}}\right)^{2}>1500
$$

If we assume $\left(r_{1} / r_{2}\right) \approx 5$ and $\left(L / r_{1}\right) \approx 12$, an $\alpha$ larger than 5 is needed for the end loss to be smaller than the radial loss.

\section{SUMMARY}

The possibility of plasma confinement using longitudinal invariant in linear devices was studied. It has a longer confinement time limited by collisions than a $\mu$-mirror. A further study is needed to test other loss processes.

\section{REFERENCE}

I. M. N. Rosenbluth, Phys. Fluids II, 869 (1968). 